\title{
Different Types of Surface Modification used for Improving the Adhesion and Interactions of Skin Cells
}

\author{
Anna Sobiepanek ${ }^{1 *}$, Joanna Baran², Małgorzata Milner-Krawczyk ${ }^{2}$ and Tomasz Kobiela ${ }^{1}$ \\ ${ }^{1}$ Laboratory of Biomolecular Interactions Studies, Chair of Drug and Cosmetics Biotechnology, Faculty of Chemistry, Warsaw \\ University of Technology, Poland
}

${ }^{2}$ Laboratory of Medical and Cosmetic Biology, Chair of Drug and Cosmetics Biotechnology, Faculty of Chemistry, Warsaw University of Technology, Poland

\begin{abstract}
Mammalian cells in the traditional 2D culture lack the complexity of 3D tissue architecture as well as the cell-cell or cell-extracellular matrix (ECM) interactions, which are present in the body. Still they are one of the most accessible and popular models used in biomedical research. Simultaneously, the understanding of various cell interactions in the body brings a valuable source of knowledge for the continuous improvement of several research areas including new biocompatible materials, tissue regeneration attempts, devices of the Lab-on-a-Chip type or cell-based biosensors for different applications. In order to meet the needs of cell culture improvement and a better understanding of the physiological cell interactions, within years different cellular surface modifications with biomolecules or chemicals have been introduced. These modifications play an important role in the regulation of several processes in cells such as adhesion, proliferation, differentiation, interaction and signalling.

In this review, we have described the typical surface modifications used for the cell culture of different skin cells with a brief specification of the mechanism of cell adhesion. The skin cells like keratinocytes, fibroblasts, melanocytes, but also cancer cells (especially melanoma cells) are the perfect models for studying adhesion mechanisms in vitro due to their mutual physiological interaction in the skin tissue.
\end{abstract}

KEYWORDS: Surface modification; Skin cells; Adhesion; Interaction; Coatings; ECM; Polymers; Chemicals

\section{INTRODUCTION}

The main skin cell types present in the epidermis are keratinocytes (main component), melanocytes (producing melanin), the Langerhans cells (dendritic cells of the immune system) and the Merkel cells (cells of the nervous system). In case of the dermis, the main cells are fibroblasts (responsible to produce growth factors) and mast cells (cells of the immune system), as well as for hypodermis - adipocytes (cells forming the adipose tissue) $[1,2]$. Due to the nature of this tissue as a continuous structure, the interactions present between several skin cell types.

Well-known fact for example, each melanocyte binds with a specified group of keratinocytes through the adhesion molecule E-cadherin to transfer melanosomes (granules of melanin), which
Quick Response Code:

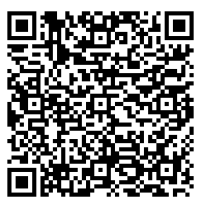

Address for correspondence: Anna Sobiepanek, LBIS, Faculty of Chemistry, Warsaw University of Technology, Noakowskiego 3, 00-664 Warsaw, Poland, Email: asobiepanek@ch.pw.edu.pl

Received: March 26, 2020 Published: April 03, 2020

How to cite this article: Sobiepanek A, Baran J, Milner-Krawczyk M, Kobiela T. Different Types of Surface Modification used for Improving the Adhesion and Interactions of Skin Cells. 2020 - 2(2) OAJBS.ID.000161. DOI: 10.38125/OAJBS.000161 
are required for protecting the keratinocytes' nucleus from the solar radiation [3]. The cessation of the E-cadherin expression breaks the control that keratinocytes have over melanocytes. In consequence, the alteration in favour of the $\mathrm{N}$-cadherin appearance occurs during the epithelial-mesenchymal transition, which is typically observed in melanoma skin cancers. With this cadherinswitch the transformed melanocytes gain a new ability to interact with fibroblasts, endothelial cells or themselves. The freshly occurring interactions are beneficial for the cancerous cells due to the production of several growth factors by fibroblasts that induce melanoma cell proliferation and migration [4].

Adherent cells can be cultured on a variety of surfaces like flat materials (typically polystyrene or glass surfaces; seldom metals such as titanium, zirconium or gold)[5,6] and in 3D structures (scaffolds from polyesters, or nanoporous materials), which are important in skin reconstructions by tissue engineering $[7,8]$. The most common surface-dependent modification is the usage of proteins found in the extracellular matrix, several polymeric materials or chemicals with precisely selected functional groups $[9,10]$. By introducing a proper growth surface modification, the regulation of various important cellular processes like adhesion, proliferation and differentiation can be controlled [11]. The surface type influences strongly the dynamic process of the cells' adhesion due to the fact that it is the result of specific interactions occurring between molecules on the cell surface and their respective ligands. The surface receptors belong to five major classes: integrins, the immunoglobulin superfamily (Ig-CAMs), cadherins, selectins and the hyaluronan receptor CD44 [12]. Adhesion occurs not only between the cells and the surface but also between the adjacent cells (cell-cell adhesion). Cell adhesion can also be a source of specific signals created during the interaction with the growth factor receptors. Due to different adhesive interactions of cells with modified surfaces, the cells' phenotype can also be regulated or altered [13]. The resistance of cells to shape changes, stretching during movement or their ability to transport cytoplasmic elements depend on the cells' cytoskeleton. The concentration and structure of individual components of the cytoskeleton (microtubules, actin filaments and intermediate filaments) also determine the cells mechanical response to the environment and facilitate the cell-cell and cell-surface interactions $[14,15]$. Therefore, the cytoskeleton dynamics as well as the growth regulation depend to some extent on the interactions associated with the term cell adhesion [13].

\section{DISCUSSION}

The frequently used surface modification with the extracellular matrix proteins facilitates the attachment of cells, by recalling the natural environment present in tissues. ECM is a group of proteins that contain the Arg-Gly-Asp (RGD) sequence to which the cell transmembrane integrin receptors bind $[6,16]$. Examples of these proteins include collagen (COL), fibronectin (FN), laminin (LM) and vitronectin (VTN) [17]. There are several identified heterodimers of integrins responsible for the adhesion of cells to the ECM proteins. The $\alpha 1 \beta 1, \alpha 2 \beta 1$ and $\alpha 3 \beta 1$ integrins interact with collagen, whereas $\alpha 4 \beta 1, \alpha 5 \beta 1, \alpha v \beta 3, \alpha \operatorname{IIb} \beta 3$ and $\alpha v \beta 5$ integrins bind to fibronectin, and also $\alpha 1 \beta 1, \alpha 2 \beta 1, \alpha 3 \beta 1, \alpha v \beta 3$ and $\alpha \operatorname{IIb} \beta 3$ interact with laminin $[18,19]$. Normal cells interact with the ECM in a carefully controlled process that allows them to integrate [20]. If detached from the extracellular matrix, normal cells are enclosed in the G1 phase of the cell cycle. Due to the loss of activity of the cyclinE/cdk2 complex, they undergo apoptosis [13]. On the other hand, during cancer transformation the binding of the integrin receptor to COL-I,
FN or VTN may lead to the matrix metalloproteinases (MMPs) production and their activation. The degradation of ECM proteins by the activated MMPs also promotes the release of the growth factors, cytokines and proteases into the environment. The growth of normal cells is inhibited and the transformed cells, in which integrin-mediated signaling pathways are disturbed, acquire the ability to grow in suspension and survive to invade other parts of the body [20].

The loss of these interactions, due to the disturbancesin adhesion molecule functions (e.g. N-CAM), may result in the uncontrolled cancer cell proliferation [13]. Moreover, surface modification with type I collagen can regulate various proliferative and survival signaling pathways of melanoma including the ephrinA1/EphA2 pathways [19]. However, metastatic melanoma cells reveal more diverse adhesion properties (from not adherent to highly adherent) to ECM proteins in comparison with the primary melanoma cells [21]. Surface modification with fibronectin may also significantly influence the melanoma cells' elastic properties like the resistance to deformation measured by the atomic force microscopy (AFM) [22]. With fibronectin adsorption onto surfaces, two protein states were identified: one with a more active conformation on the hydrophilic surface and the other less active on the hydrophobic. In case of hydrophobic materials, the hydrophobic interactions are stronger which leads to the rearrangement of the secondary structure of FN. Consequently, fewer fibroblasts have bound and spread on hydrophobic and more on the hydrophilic surfaces. In addition to wettability, functional groups and surface charges also affect protein adsorption, and thus cell adhesion [23].

The analogic type of surface modification is provided by the use of Matrigel, the mixture consisting of various concentrations of ECM proteins, which has been extracted from Engelbreth-HolmSwarm tumors. Its use should allow reconstituting the basement membrane. However, Matrigel is not a well-defined matrix and because of that it can be responsible for the differences in the experimental results [24]. Moreover, the RGD peptide sequence alone can be covalently attached to several surfaces and scaffolds. The amino acid order in the peptide is important as confirmed by the study of fibroblast adhesion and their spreading on surfaces modified with analogical sequences. The RGD (Arg-Gly-Asp) sequence partially inoculated in the interpenetrating networks of the acrylic acid copolymer and polyethylene glycol (PEG) promoted fibroblast spreading in contrast to the polymer films containing RDG (Arg-Asp-Gly) or RGE (Arg-Gly-Glu) sequence motif. Similarly, hydrogels made of PEG containing RGD promoted the spread of fibroblasts, whereas those containing RDG did not [25]. The weakness of these modifications can relate to a possibility of the ECM degradation due to microenvironmental changes (e.g. $\mathrm{pH}$ ) or the protein digestion by the bound cells [26].

Using the ECM coating for cell seeding is also quite popular in several procedures of the skin cell isolation from the tissue. Especially in case, when the tissue fragment was exposed to the enzymatic digestion or the mechanical disaggregation $[7,27,28]$. However, the surface modification for improving cell adhesion can be performed for several types of materials, not only glass or polystyrene. For the tissue regeneration attempts, biodegradable synthetic polymers (like polylactide, polycaprolactone or polylactide-co-glycolide (PLGA)) can be formed into nanofibers by the electrospinning process. They provide stable mechanical support for cells, but at the same time these polymers are unable to provide sufficient support for cellular adhesion, which is why 
surface modification with ECM proteins is required. For example, the fibrin coating significantly improved the attachment, spreading and proliferation of fibroblasts, whereas collagen coating had a positive influence on the behavior of keratinocytes. This is why the nanofibrous membranes with protein nanocoating are considered as promising constructions of the skin substitute [29]. Similar observations were performed with the COL and FN surface modification of the nanoporous anodic alumina (NAA) and porous silicon (PSi). These structural biomaterials after their functionalization with ECM proteins have good adhesive properties for cells [8].

Following the cell adhesion process on different surfaces in real-time is possible by means of advanced methods like the quartz crystal microbalance with the dissipation monitoring (QCM-D) $[18,30]$. Not only does this method provide information about the changes in the frequency shift connected with the increase of the mass bound to the surface of the sensor, but also of the viscoelastic changes of the newly created layer [31]. However, for experiments on cell adhesion the surface of the sensor (typically gold) has to be pre-coated with the studied protein before the measurement starts [32]. For example, with the QCM-D method significant differences in the adhesion of melanoma cells were investigated on the FN and VTN pre-coated sensors. The cells have attached strongly to the vitronectin coating and after 1.5 hours the rearrangement in the cells began, whereas in case of laminin coating the observed changes in the frequency shift were probably connected with the partial degradation of LM by cells [33].

Another possibility for the modification of various surfaces is the application of chemical reagents including cationic polyamino acids like poly(lysine) (PL), poly(ornithine) (PO), poly(arginine) and poly(ethylenimine) (PEI). These positively charged polymers facilitate cell adhesion through the attraction of the negatively charged cell membrane. They are usually deposited from $0.01 \%$ solution to the modified surface and depending on their properties like chirality (e.g. poly-L-lysine (PLL), poly-D-lysine (PDL)) and molecular weight they may have different modes of actions and toxicity towards various cell types [26]. The optimization of the cell culture conditions is a very important issue concerning these coatings because of their effect on the cell proliferation rate, attachment, morphology and also the cellular cytoskeleton rearrangement [34]. This type of surface modification is rather resistant to degradation, however, cell types that secrete proteases are able to digest poly-L-lysine more easily than poly-D-lysine [26].

Metal surfaces like gold can be coated with polystyrene $(0.5 \%$ PS solution in toluene) and the following exposure of the PS surface to the ultraviolet light increases its wettability facilitating cell adhesion $[35,36]$. To increase the cell adsorption to the PS surface, the optional sulfonation with the concentrated sulfuric acid can be performed, which increases the number of charged groups on the surface [37]. Another method of PS modification to increase cell adhesion is to treat the surface with chromic acid, which leads to the formation of aldehyde or carboxyl groups on the PS surface [38]. Common gold modifications are also those performed with the use of the self-assembling monolayers (SAMs) like thiols and siloxanes. This can be made either through the covalent conjugation or by the physical adsorption of these compounds to gold. In practice, SAM preparation usually involves a direct immersion of a metal to be modified in a diluted solution of a suitable modifying compound (e.g. gold in a thiol solution). Through this method layers of small thickness (about 1-3 nm) are obtained [39]. Thiol-containing polyethylene glycol (thiol-PEG) coating is believed to be non-toxic for cells [40] and at the same time effective at limiting non-specific adsorption of proteins to gold surfaces [41].

Such anti-fouling behavior is mainly due to the steric repulsion between hydrated neutral PEG chains and proteins [23]. Polydimethylsiloxane (PDMS) has a number of features and properties that allow its use for the construction of Lab-on-a Chip microsystems in which adherent cells can be grown and treated. These include biocompatibility, high gas permeability ensuring oxygen delivery to the cells in microchannels, transparency as well as stability over a wide range of temperatures [42]. The ability of cell interaction with PDMS is determined by the surface properties such as the chemical composition of the obtained layer, its hydrophobicity or hydrophilicity, as well as the topography and stiffness of the material. Furthermore, the parameters for the surface modification with PDMS must be properly selected due to the fact that different substratum stiffness (0.75 $\mathrm{MPa}-2.92 \mathrm{MPa})$ significantly influences the cell morphology and its proliferation rate [43]. These parameters are the incubation time, temperature and compound concentration. Also, parameters related to the cell culture on these surfaces (e.g. culture time, the composition of the culture medium) are important in each cell adhesion experiment.

\section{CONCLUSION}

The type of growth surface has a crucial effect on the cells' binding to the substrate, due to the fact that it may force cells to change the adhesion mechanism. With the surface modification, it is possible to control the behavior of the cells, to increase the adhesion of one type of cell and limit the growth of the other types of cells. However, it is also desirable that the applied surface modification would simplify the binding of the cells to the surface and generally enable their good spreading. Some of the crucial parameters before surface modification which should be considered are the composition, orientation, hydrophilicity and viscoelasticity of the selected material for cell adhesion. Therefore, the biophysical characteristics of the living cell interaction with modified surfaces brings much useful information, especially in the context of several diseases including cancer.

\section{ACKNOWLEDGMENT}

This work was supported by the National Science Centre (Poland) grant no. 2017/27/N/ST4/01389 and by the Warsaw University of Technology.

\section{ORCID}

Anna Sobiepanek https://orcid.org/0000-0003-3186-7582

Joanna Baran https://orcid.org/0000-0001-8916-6020

Małgorzata Milner-Krawczyk https://orcid.org/0000-00032032-0995

Tomasz Kobiela https://orcid.org/0000-0001-7155-659X

\section{REFERENCES}

1. De Falco M, Pisano MM, De Luca A (2014) Embryology and anatomy of the skin. In Baldi A, Pasquali P, Spugnini EP (eds) Skin cancer: A practical approach, Springer, New York, USA.

2. Graham HK, Eckersley A, Ozols M, Mellody KT, Sherratt MJ (2019) Human skin: Composition, structure and visualisation methods. In Limbert G (eds) Skin biophysics. Studies in mechanobiology, tissue engineering and biomaterials, Springer International Publishing, New York, USA. 
3. Ando H (2012) Melanosomes are transferred from melanocytes to keratinocytes through the processes of packaging, release, uptake and dispersion. J Inves Dermat 132(4):1222-1229.

4. Marczyńska D, Przybyło M (2013) Melanocyty - komórki barwnikowe o wielu obliczach. Kosmos 62(4): 491-499.

5. Depprich R (2008) Behavior of osteoblastic cells cultured on titanium and structured zirconia surfaces. Head \& Face Medicine 4: 29.

6. Sobiepanek A, Kobiela T (2018) Application of biosensors in cancer research. Review and Research on Cancer Treatment 4(1): 4-12.

7. Moulin VJ (2011) In vitro culture methods of skin cells for optimal skin reconstruction by tissue engineering. In Eberli D (eds) Regenerative medicine and tissue engineering-cells and biomaterials, In tech. USA.

8. Formentín P (2018) Collagen and fibronectin surface modification of nanoporous anodic alumina and macroporous silicon for endothelial cell cultures. J Biolog Engin12(1): 21.

9. Ryan JA (2008) Evolution of cell culture surfaces. Bio Files 3: 8-11.

10. Teramura Y (2017) Cell adhesion induced using surface modification with cell-penetrating peptide-conjugated poly (ethylene glycol)-lipid: A new cell glue for $3 \mathrm{~d}$ cell-based structures. ACS Applied Materials \& Interfaces 9(1): 244-254.

11. Wang JL (2013) Direct adhesion of endothelial cells to bioinspired poly(dopamine) coating through endogenous fibronectin and integrin A5 B1. Macromol Biosci 13(4): 483-493.

12. Makrilia N, Kollias A, Manolopoulos L, Syrigos K (2009) Cell adhesion molecules: role and clinical significance in cancer. Cancer Invest 27(10): 1023-1037.

13. Rusciano D (2011) Adhesion. In: Schwab M (eds) Encyclopedia of cancer. Springer, Berlin, New York, USA.

14. Fletcher DA, Mullins RD (2010) Cell mechanics and the cytoskeleton. Nature 463(7280): 485-492.

15. Sobiepanek A, Milner-Krawczyk M, Bobecka-Wesołowska K, Kobiela $\mathrm{T}$ (2016) The effect of delphinidin on the mechanical properties of keratinocytes exposed to UV radiation. Journal of Photochemistry and Photobiology Biology 164: 264-270.

16. Marcotte L, Tabrizian M (2008) Sensing surfaces: Challenges in studying the cell adhesion process and the cell adhesion forces on biomaterials. IRBM 29(2-3): 77-88.

17. Ahmed N, Clyde R, Greg R, Michael Q (2005) Role of integrin receptors for fibronectin, collagen and laminin in the regulation of ovarian carcinoma functions in response to a matrix microenvironment. Clin Exp Metastasis 22(5): 391-402.

18. Kramer RH, Vu M, Cheng YF, Ramos DM (1991) Integrin expression in malignant melanoma. Cancer Metastasis Rev 10(1):49-59.

19. Aoudjit F, Vuori K (2012) Integrin signalling in cancer cell survival and chemoresistance. Chemother Res Pract 2012: 1-16.

20. Huh SJ (2010) KLF6 gene and early melanoma development in a collagen I-rich extracellular environment. J Natl Cancer Inst 102(15): 1131-1147.

21. Laidler $P$ (2006) Characterization of glycosylation and adherent properties of melanoma cell lines. Cancer Immunology, Immunotherapy 55(1): 112-118.

22. Weder G (2014) Increased plasticity of the stiffness of melanoma cells correlates with their acquisition of metastatic properties. Nanomedicine: Nanotechnology, Biology and Medicine 10(1): 141-148.

23. Schulte VA, Díez M, Möller M, Lensen MC (2009) Surface topography induces fibroblast adhesion on intrinsically nonadhesive poly (Ethylene Glycol) substrates. Biomacromolecules 10(10): 2795-2801.

24. Hughes CS, Postovit LM, Lajoie GA (2010) Matrigel: A complex protein mixture required for optimal growth of cell culture. PROTEOMICS 10(9): $1886-1890$.
25. Mann BK, West JL (2002) Cell adhesion peptides alter smooth muscle cell adhesion, proliferation, migration, and matrix protein synthesis on modified surfaces and in polymer scaffolds. J Biomed Mater Res 60(1): 86-93.

26. Blau A (2013) Cell adhesion promotion strategies for signal transduction enhancement in microelectrode array in vitro electrophysiology: an introductory overview and critical discussion. Current Opinion in Colloid \& Interface Science 18(5): 481-492.

27. Tsuji T, Karasek M (1983) A procedure for the isolation of primary cultures of melanocytes from newborn and adult human skin. J Invest Dermatol 81(2): 179-180.

28. Perez DE (2018) Uveal melanoma driver mutations in GNAQ/11 yield numerous changes in melanocyte biology. Pigment Cell \& Melanoma Research 31(5): 604-613.

29. Bacakova M (2017) Protein nanocoatings on synthetic polymeric nanofibrous membranes designed as carriers for skin cells. Int J Nanomedicine 9(12): 1143-1160.

30. Kılıç A, Kok FN (2018) Quartz crystal microbalance with dissipation as a biosensing platform to evaluate cell-surface interactions of osteoblast cells. Biointerphases 13(1): 011001.

31. Sobiepanek A, Milner-Krawczyk M, Lekka M, Kobiela T (2017) AFM and QCM-D as tools for the distinction of melanoma cells with a different metastatic potential. Biosensors and Bioelectronics 93: 274-81.

32. Marx KA (2003) Quartz crystal microbalance: a useful tool for studying thin polymer films and complex biomolecular systems at the solutionsurface interface. Biomacromolecules 4(5): 1099-1120.

33. Fohlerová Z, Skládal P, Turánek J (2007) Adhesion of eukaryotic cell lines on the gold surface modified with extracellular matrix proteins monitored by the piezoelectric sensor. Biosensors and Bioelectronics 22(9-10): 1896-1901.

34. Liberio MS, Sadowski MC, Soekmadji C, Davis RA, Nelson CC (2014) Differential effects of tissue culture coating substrates on prostate cancer cell adherence, morphology and behaviour. PLoS ONE 9(11): e112122.

35. Pei Z (2012) Real-time analysis of the carbohydrates on cell surfaces using a qcm biosensor: a lectin-based approach. Biosensors and Bioelectronics 35(1): 200-205.

36. Peiris D, Markiv A, Curley GP, Dwek MV (2012) A novel approach to determining the affinity of protein-carbohydrate interactions employing adherent cancer cells grown on a biosensor surface. Biosens Bioelectron 35(1): 160-166.

37. Martin GR, Rubin H (1974) Effects of cell adhesion to the substratum on the growth of chick embryo fibroblasts. Experimental Cell Research 85(2): 319-333.

38. Curtis AS, Forrester JV, McInnes C, Lawrie F (1983) Adhesion of cells to polystyrene surfaces. J Cell Biol 97(5): 1500-1506.

39. Ulman A (1996) Formation and structure of self-assembled monolayers. Chemical Reviews 96(4): 1533-1554.

40. Manohar S, Rayavarapu R, Petersen W, van Leeuwen TG (2009) Cell viability studies of PEG-thiol treated gold nanorods as optoacoustic contrast agents. Proceedings of SPIE - The International Society for Optical Engineering 7177.

41. Tan YH (2013) Lectin-carbohydrate interactions on nanoporous gold monoliths. New Journal of Chemistry 37(7): 2150.

42. Temiz Y, Lovchik RD, Kaigala GV, Delamarche E (2015) Lab-on-a-chip devices: How to close and plug the lab?. Microelectronic Engineering 132: $156-175$.

43. Prauzner-Bechcicki S (2015) PDMS substrate stiffness affects the morphology and growth profiles of cancerous prostate and melanoma cells. J Mech Behav Biomed Mater 41(Suppl C): 13-22. 